\title{
Real-time Identification of Guidance Modes in Aircraft Descents Using Surveillace Data
}

\author{
An Interacting Multiple-model (IMM) filter application \\ Ramon Dalmau, Marc Pérez-Batlle and Xavier Prats \\ Department of Physics - Aeronautics Division \\ Technical University of Catalonia - BarcelonaTECH \\ Castelldefels, Spain
}

\begin{abstract}
Trajectory predictors require information on the flight-intent in order to estimate the future state of the aircraft. At present, however, such information is not available or it is very limited and coarse (unless predicting the ownship trajectory). In this paper, an interacting multiple-model (IMM) algorithm is proposed to improve the accuracy of short-term trajectory predictions. The active guidance mode of an aircraft is estimated in real-time observing flight data collected only from automatic dependent surveillance-Broadcast (ADS-B) and transponder selective mode (Mode S) emissions. The algorithm is set up with different models corresponding to the most typical guidance modes, and provides the model that better fits the observations. The proposed algorithm is validated by means of two simulated trajectories whose guidance modes were known beforehand. Finally, the performance of the algorithm with real flight data is demonstrated through a detailed example. Promising results are obtained, showing that the active guidance mode can be unequivocally identified with a negligible delay.

Index Terms - Trajectory prediction, Guidance modes, Kalman filter, ADS-B, interacting multiple-model
\end{abstract}

\section{INTRODUCTION}

The future Air Traffic Management (ATM) system will move towards a trajectory centric paradigm, aiming at the same time, to optimise safe air traffic flow at more strategic stages. A key aspect for this new concept of operations is the capability to guarantee common situational awareness among relevant stakeholders, as a function of time extrapolated into the future, with decision support tools making use of advanced trajectory predictors (TP) and conflict detection and resolution (CDR) algorithms.

TPs are needed for a wide variety of applications: onboard trajectory planning and guidance, flow management, air traffic control (ATC), sequencing and merging, on-board or ground separation management, on-board or ground collision avoidance, etc. In [1], a comprehensive review of TP and CDR applications is given and discusses the key performance drivers that underline the TP and CDR performance requirements. A TP estimates the most likely trajectory of an aircraft given its observed current state; a weather forecast; and a flight-intent. CDR capabilities, in turn, are underpinned by the sharing of the instantaneous and predicted aircraft positions, which are provided by navigation systems and TP algorithms.
Accurate weather data is currently available from stateof-the-art numerical weather prediction models, which can be used for ground applications or eventually, up-linked to aircraft. Moreover, the strategy of sharing ownship measured meteorological data with surrounding aircraft could also lead to a significant enhancement of the quality of the weather data handled by on-board TPs [2], [3].

In general, an accurate knowledge of aircraft performance data and flight-intent is available for ownship TP algorithms. However, on-board applications for intruder trajectory prediction, to enable for instance self-separation or conformance monitoring applications [4], rely on simplified aircraft performance data and have a very limited (or non-existent) knowledge of the intruder's flight-intent. A similar limitation is found for ground-based TPs, which typically use the Airline Procedure Model (ARPM), embedded in the Base of Aircraft Data (BADA) [5]. The ARPM, however, tends to be too generalist for most applications [6].

Good flight-intent data is specially critical in the vertical domain, since slight input inaccuracies easily lead to big discrepancies in the vertical (and speed) trajectory profile that is finally computed by the TP. Aircraft vertical intent is composed by a sequence of flight phases with different parametrised guidance modes (e.g. descent at constant Mach and idle thrust) and end conditions (e.g. until reaching the target altitude). These guidance modes describe how the throttle and elevator will operate to follow the planned the trajectory, and must be known by the TP to integrate the equations describing the aircraft dynamics.

In this paper a novel methodology is presented with the objective to identify, in real time, the active guidance mode of an aircraft, using only as flight observables data from a automatic dependent surveillance-broadcast (ADS-B) and selective mode transponder (Mode $S$ ) receiver. The paper is focused in aircraft climbing or descending, which are more challenging to track and predict, and considers non-standard atmospheric conditions. A clear application of this methodology would be to provide accurate and reliable flight-intent data to any kind of airborne or ground-based TP, but would also enable a wide range of ATM performance studies, ATM analytics [6]. 
The methodology presented in this paper is based in a multiple-model (MM) algorithm, which have been widely used to track vehicles in the last decades. MM algorithms are used when a single model cannot represent the motion of a body in all its possible stages or manoeuvres. Excellent surveys of the MM fundamentals and applications can be found in [7], [8]. MM algorithms have the ability to estimate the state of a dynamic system with multiple models (in this paper representing the aircraft guidance modes), which can dynamically change in an unanticipated manner, and to identify the maximum likelihood model at each time stamp.

In [9] a MM filter, based on 2D kinematic models, was used to improve the tracking of aircraft for CDR applications. Similarly, in [10] a MM filter was developed to identify aircraft manoeuvres during taxi operations. An enhanced MM filter, using a non-linear point mas model to describe 3D aircraft dynamics, was proposed in [11]; showing significant benefits in terms of position estimation accuracy and filter robustness with respect to conventional kinematic-based filters.

All previous works, however, aimed at identifying simple manoeuvres in the horizontal plane, such as coordinated turns, constant speed or acceleration segments. In [12] manoeuvres in the vertical plane were considered, but the algorithm was limited to identify simple manoeuvres such as constant altitude rate at constant speed. Up to the authors best knowledge, no research effort has been devoted to targeting the different (and complex) guidance modes that describe aircraft vertical dynamics. The method proposed in this paper aims to discriminate the maximum likelihood model from a finite set of predefined models, each one corresponding to a generic guidance mode that defines the vertical (and speed) profile of the aircraft trajectory. The performance of the algorithm with real flight data is assessed through a detailed example.

\section{BACKGROUND}

The motion of an aircraft can be accurately described with a six degree of freedom model (6DOF), which consists on a system of ordinary differential equations (ODEs) describing the three translations and the three rotations of the aircraft. Although this model results in the most accurate representation of the aircraft dynamics, it requires an extensive aerodynamic model and the knowledge of the inertia tensor of the aircraft.

Basic trajectory prediction tools use macroscopic models, where only kinematic equations are considered. Somewhere in between these two approaches, lie the aircraft point-mass models, which are considered accurate enough for on-board trajectory prediction systems and for the majority of groundbased ATM applications [13]. In this model, the aircraft motion is reduced to three degrees of freedom, assuming that all forces are applied to the centre of gravity of the aircraft.

Going further, many ATM applications reduce the pointmass model to what is typically called a gamma-command model, where continuous vertical equilibrium is assumed. This hypothesis entails to neglect the dynamics of the aerodynamic flight path angle, which is thereupon considered as an input control variable that can change instantaneously. Although many simplifications are needed to reach the gamma-command model, it is accurate enough to represent aircraft operating under the most typical guidance modes by complementing the model with two additional path constraints[14].

Multiple-model estimation algorithms could be used to identify the form of the gamma-command model plus path constraints (each form representing a different guidance mode) that better fit the observations. The MM approach is based on the fact that the behaviour of a "real" system cannot be characterised at all times by the same model. Instead, a finite number of models should be considered to adequately describe its behaviour in all possible manoeuvres and stages.

Typical algorithms for state and model estimation based on MM consist of a set of elementary filters (each one associated with a particular model) and a certain algorithm to manage the cooperation among them. MM can be set up using Kalman filters (KF) as elementary filters, or extended Kalman filters (EKF) to account for non-linearities in the motion and/or measurements models. A particular MM algorithm that achieves an excellent compromise between performance and complexity is the interacting multiple model (IMM) [15].

Section II-A) presents the gamma-command model and describes the generic guidance modes to operate an aircraft in the vertical plane. Section II-B presents the mathematical background of the IMM algorithm implemented in this paper.

\section{A. Aircraft dynamics model}

The motion of the aircraft in the vertical plane can be described by the following system of ODEs

$$
\begin{aligned}
\frac{\mathrm{d} v}{\mathrm{~d} t} & =\dot{v}=\frac{T-D}{m}-g \sin \gamma \\
\frac{\mathrm{d} h}{\mathrm{~d} t} & =\dot{h}=v \sin \gamma \\
\frac{\mathrm{d} m}{\mathrm{~d} t} & =\dot{m}=-F F
\end{aligned}
$$

where the state vector $\boldsymbol{x}=[v, h, m]$ is composed by the true airspeed (TAS), the geometric altitude and the mass of the aircraft; the generic control vector of this model $\boldsymbol{u}=[T, \gamma]$ is composed by the engine thrust and the aerodynamic flight path angle (FPA); and $D$ is the aerodynamic drag, $g$ the gravity acceleration and $F F$ the engine fuel flow.

In order to integrate Eq. (1) two degrees of freedom must be closed. In other words, the aircraft guidance system uses two independent actuators to steer the aircraft along the vertical plane: the elevator and the throttle. In the remainder of this paper it is convened that the $1^{\text {st }}$ degree of freedom corresponds to the elevator and the $2^{\text {nd }}$ is associated to the throttle setting.

Mathematically, the elevator and the throttle could directly control $\gamma$ and $T$, respectively, and easily close the two degrees of freedom of Eq. (1). In practise, however, aircraft are not operated following specific $T$ and $\gamma$ profiles, and these control variables are not known beforehand. Instead, climbs and descents are typically composed by constant Mach or callibrated airspeed (CAS) segments, while in the cruise phase aircraft fly at constant pressure altitude and Mach, for instance. 
In a more generic formulation, two path constraints $(c)$ close Eq. (1), rather than a given control vector:

$$
c_{i}(\boldsymbol{x}, \boldsymbol{u}, \boldsymbol{p})=0 ; \quad i \in\{1,2\} .
$$

where $\boldsymbol{p}$ is a vector of parameters.

Equations (1) and (2) together form a system of differentialalgebraic equations (DAEs) that fully describe a trajectory in the vertical plane. Unless $\gamma$ and $T$ are given as an input control (rare case in operational situations), it will be always needed to compute them in order to transform the original set of DAEs to a system of ODEs suitable for numerical integration.

Some phases of flight are typically flown at a given throttle (such as idle, maximum climb, etc.). In such case, the second degree of freedom would be closed by the following path constraint, from which the thrust can be directly derived:

$$
T-T_{i d l e}-\pi\left(T_{\max }-T_{i d l e}\right)=0 ; \quad \pi \in[0,1]
$$

where maximum and the minimum thrust ( $T_{\max }$ and $\left.T_{i d l e}\right)$, aircraft-dependent functions of the true airspeed (TAS) and the temperature and pressure of the air ( $\tau$ and $p$, respectively). Under these conditions, the elevator would close the first degree of freedom by either controlling the ground FPA, the vertical speed (VS), or by following a certain speed profile.

1) Fixed throttle setting and ground FPA: A trivial case where the aerodynamic FPA (control) equals to the desired ground FPA (parameter). Thus the path constraint is:

$$
\gamma-\gamma_{g}=0
$$

In Eq. (4) the curvature of the earth and the cross and alongpath wind components have been neglected.

2) Fixed throttle setting and vertical speed: It should be noted that the operational vertical speed (VS) is the rate of change of the pressure altitude $\left(h_{p}\right)$, and not the geometrical altitude. VS and $\dot{h}$ are related through the temperature of the air and its the deviation with respect to the international standard atmosphere (ISA) as follows:

$$
\mathrm{VS}=\frac{\tau-\Delta \tau}{\tau} \frac{\mathrm{d} h}{\mathrm{~d} t}
$$

If $\mathrm{VS}$ is given as guidance parameter, then the path constraint associated with the elevator would directly define the required aerodynamic FPA as follows:

$$
\gamma-\arcsin \left(\frac{\mathrm{VS}}{v} \frac{\tau}{\tau-\Delta \tau}\right)=0
$$

3) Fixed throttle setting and energy share factor: When the speed profile is controlled in a climb/descent (e.g. constant Mach or CAS), typical guidance systems use the energy share factor $(k)$, which specifies how much of the excess power is allocated to gain potential energy, at the expense of kinetic energy in such a way the speed profile is followed.

$$
k=\left(1+\frac{v}{g} \frac{\mathrm{d} v}{\mathrm{~d} h}\right)^{-1}
$$

If the throttle controls the amount of thrust by enforcing the second path constraint (3), the path constraint associated with the elevator defines the flight path angle required to accomplish a certain combination of $k$ and $\pi$ :

$$
\gamma-\arcsin \left(\left(\frac{T-D}{m g}\right) k\right)=0
$$

Equation (8) is derived by combining Eqs. (1a),(1b) and (7).

4) Variable throttle setting: Conversely, for those guidance modes in which the elevator controls either VS or the geometric FPA and the throttle manages the speed, the path constraint associated with the throttle is defined by Eq. (8) rather than (3), where $\gamma$ should be substituted by either Eq. (4) or Eq. (6), depending on the variable controlled by the elevator.

Summing up, in this paper the path constraints that can close the mathematical problem are given by Eqs. (3),(4),(6) and (8) and the parameters vector is composed by $\boldsymbol{p}=\left[\pi, \mathrm{VS}, \gamma_{g}, k\right]$. Each guidance mode will be defined by a combination two of these constraints and will be parameterised in terms of $\boldsymbol{p}$.

\section{B. Interacting multiple model extended Kalman filter}

Fundamentally, the goal of a filtering algorithm is to provide the best estimate of the current state of a system, based on the state at the previous time step(s); a model describing the state dynamics with a certain confidence; and measurements, which are typically corrupted with some amount of error.

It is well known that the $\mathrm{KF}$ is the optimal state estimator for linear, unconstrained systems in presence of normally distributed state and measurement noises. However, the dynamics of an aircraft described by the point-mass model given by Eq. (1) are non-linear. Therefore, the traditional KF cannot be directly applied to estimate the state of such a system.

The EKF extends the scope of the conventional KF to nonlinear optimal filtering problems by generating a Gaussian approximation to the joint distribution of the state and measurements vectors using a Taylor series expansion[16]:

$$
\begin{aligned}
\boldsymbol{x}_{k} & =\boldsymbol{f}\left(\boldsymbol{x}_{k-1}, \boldsymbol{u}_{k-1}, \boldsymbol{p}\right)+\boldsymbol{q}_{k-1} \\
\boldsymbol{y}_{k} & =\boldsymbol{h}\left(\boldsymbol{x}_{k}, \boldsymbol{p}\right)+\boldsymbol{r}_{k}
\end{aligned}
$$

where $\boldsymbol{y}$ is the measurement vector; $\boldsymbol{q} \sim \mathcal{N}(0 ; \boldsymbol{Q})$ is the process noise; $\boldsymbol{r} \sim \mathcal{N}(0 ; \boldsymbol{R})$ is the measurement noise; $\boldsymbol{f}$ and $\boldsymbol{h}$ are the dynamics and measurement model function vectors, respectively. $\boldsymbol{Q}$ and $\boldsymbol{R}$ are a priori known matrix representing the variance of the process and measurements, respectively. The initial state is a random vector with a known expected value $\boldsymbol{x}_{0}$ and covariance $\boldsymbol{P}_{0}$.

The EKF filtering process is separated into two major steps: the prediction and the update. Given the estimated expected value and covariance of the state vector at a given time instant ( $\hat{\boldsymbol{x}}$ and $\boldsymbol{P}$, respectively), a rough prediction of these values after a time step can be performed with $f$ as follows:

$$
\begin{aligned}
\boldsymbol{x}_{k}^{-} & =\boldsymbol{f}\left(\hat{\boldsymbol{x}}_{k-1}, \boldsymbol{u}_{k-1}, \boldsymbol{p}\right) \\
\boldsymbol{P}_{k}^{-} & =\boldsymbol{F}_{x}\left(\hat{\boldsymbol{x}}_{k-1}, \boldsymbol{u}_{k-1}, \boldsymbol{p}\right) \boldsymbol{P}_{k-1} \boldsymbol{F}_{x}^{T}\left(\hat{\boldsymbol{x}}_{k-1}, \boldsymbol{u}_{k-1}, \boldsymbol{p}\right)+\boldsymbol{Q}_{k-1} .
\end{aligned}
$$


where $\boldsymbol{x}^{-}$and $\boldsymbol{P}^{-}$denote the predicted expected value and covariance of the state, respectively, before acquiring the measurement; $\boldsymbol{F}_{x}$ and $\boldsymbol{H}_{x}$ are the Jacobians of $\boldsymbol{f}$ and $\boldsymbol{h}$, respectively. During the EKF update, the predictions are combined with the most recent measurements to provide a better estimate. In order to accomplish that, $\boldsymbol{x}^{-}$and $\boldsymbol{P}^{-}$are updated using a weighted average with the latest measurements:

$$
\begin{aligned}
\boldsymbol{v}_{k} & =\boldsymbol{y}_{k}-\boldsymbol{h}\left(\boldsymbol{x}_{k}^{-}, \boldsymbol{u}_{k}, \boldsymbol{p}\right) \\
\boldsymbol{S}_{k} & =\boldsymbol{H}_{x}\left(\boldsymbol{x}_{k}^{-}, \boldsymbol{u}_{k}, \boldsymbol{p}\right) \boldsymbol{P}_{k}^{-} \boldsymbol{H}_{x}^{T}\left(\boldsymbol{x}_{k}^{-}, \boldsymbol{u}_{k}, \boldsymbol{p}\right)+\boldsymbol{R}_{k} \\
\boldsymbol{K}_{k} & =\boldsymbol{P}_{k}^{-} \boldsymbol{H}_{x}^{T}\left(\boldsymbol{x}_{k}^{-}, \boldsymbol{u}_{k}, \boldsymbol{p}\right) \boldsymbol{S}_{k}^{-1} \\
\hat{\boldsymbol{x}}_{k} & =\boldsymbol{x}_{k}^{-}+\boldsymbol{K}_{k} \boldsymbol{v}_{k} \\
\boldsymbol{P}_{k} & =\boldsymbol{P}_{k}^{-}-\boldsymbol{K}_{k} \boldsymbol{S}_{k} \boldsymbol{K}_{k}^{T}
\end{aligned}
$$

where $\boldsymbol{v}$ is the innovation; $\boldsymbol{S}$ the measurement prediction covariance; and $\boldsymbol{K}$ the Kalman gain, which indicates how much the estimates from the prediction step should be corrected.

In many practical scenarios it is reasonable to postulate that Eq. (9) can change through time. In such cases, a single model cannot accurately describe the real system.

The basic idea of the EKF-IMM is to assume a fixed set $\mathcal{M}$ of $N$ models as possible candidates of the "true" model at each time step instant. Each model $\mathrm{M}^{j}$ is associated with a different elementary EKF which performs its own estimation. The overall state expected value and process covariance estimates are computed based on the results of these elementary filters and their corresponding probabilities of being the true model.

Each model $\mathrm{M}^{j}$ has some known prior probability $\mu_{0}^{j}=$ $P\left\{\mathrm{M}_{0}^{j}\right\}$. In addition, the probabilities of switching from model $i$ to model $j$ in the following time step $\left(p_{i j}=\right.$ $P\left\{\mathbf{M}_{k}^{j} \mid \mathbf{M}_{k-1}^{i}\right\}$ ) are also assumed to be known.

The optimal approach to filter the states of multiple model systems consists of three major steps: interaction (mixing); filtering; and combination (see the workflow of Fig. 1). Next, the mathematical details of each particular step are presented.

1) Interaction step: First, the initial conditions for each elementary EKF are obtained by mixing the estimates produced by all filters from the previous time step under the assumption that the concerned model is the right model at the current time step. The mixing probabilities $\mu_{k}^{i \mid j}$ for each pair of models $i$ and $j$ are calculated as follows:

$$
\bar{c}_{j}=\sum_{i=1}^{N} p_{i j} \mu_{k-1}^{i} ; \quad \mu_{k}^{i \mid j}=\frac{1}{\bar{c}_{j}} p_{i j} \mu_{k-1}^{i}
$$

where $\mu^{i}$ is the probability of model $i$ and $\bar{c}_{j}$ is a normalisation factor. Then, the mixed inputs for each filter are computed as:

$$
\begin{aligned}
\hat{\boldsymbol{x}}_{k-1}^{0 j} & =\sum_{i=1}^{N} \mu_{k}^{i \mid j} \hat{\boldsymbol{x}}_{k-1}^{i} \\
\boldsymbol{P}_{k-1}^{0 j} & =\sum_{i=1}^{N} \mu_{k}^{i \mid j} \boldsymbol{P}_{k-1}^{i}\left(\hat{\boldsymbol{x}}_{k-1}^{i}-\hat{\boldsymbol{x}}_{k-1}^{0 j}\right)\left(\hat{\boldsymbol{x}}_{k-1}^{i}-\hat{\boldsymbol{x}}_{k-1}^{0 j}\right)^{T}
\end{aligned}
$$

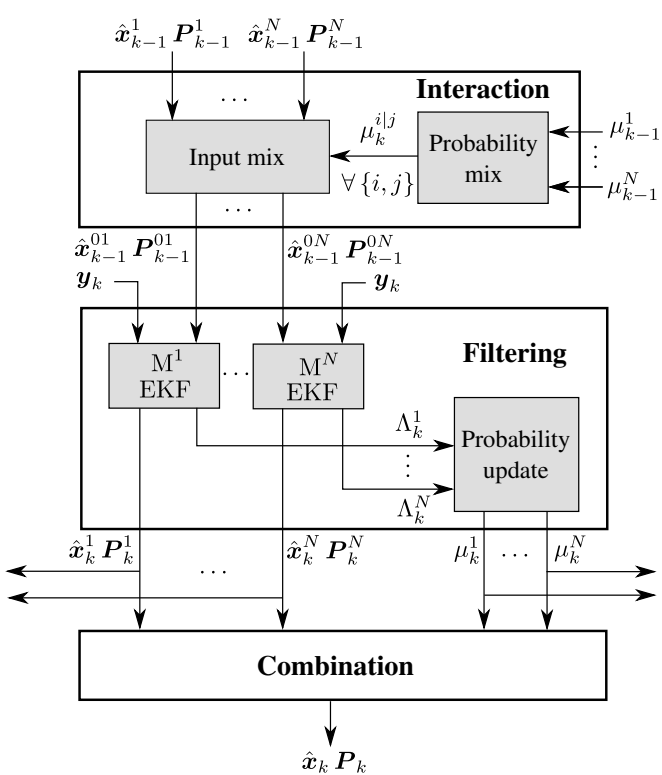

Fig. 1. Interacting multiple-model (IMM) flow diagram

where $\hat{\boldsymbol{x}}^{0 j}$ and $\boldsymbol{P}^{0 j}$ are the mixed expected values and covariances of model $j$, used to initialise the filtering step.

2) Filtering step: The filtering step of the EKF-IMM consists on performing a standard EKF step for each elementary filter. That is, both prediction and update steps are performed to obtain the expected value and covariance for each modelmatched filter $i$ at time step $k$ ( $\boldsymbol{x}_{k}^{i}$ and $\boldsymbol{P}_{k}^{i}$, respectively).

In addition to the expected value and covariance, the likelihood of the measurement for each model $i$ at time step $k$ is computed as $\Lambda_{k}^{i}=\mathcal{N}\left(\boldsymbol{v}_{k}^{i} ; 0, \boldsymbol{S}_{k}^{i}\right)$; where $\boldsymbol{v}_{k}^{i}$ is the innovation and $\boldsymbol{S}_{k}^{i}$ is the covariance for model $i$ at the EKF update $k$.

As a result of the filtering step, the probabilities of each model $i$ at time step $k$ can be calculated as follows:

$$
\bar{c}=\sum_{i=1}^{N} \Lambda_{k}^{i} \bar{c}_{i} ; \quad \mu_{k}^{i}=\frac{1}{\bar{c}} \Lambda_{k}^{i} \bar{c}_{i}
$$

3) Combination step: Finally, a weighted combination of the updated expected value and covariance estimates produced by all the filters is performed to obtain the final IMM estimate for the state and covariance of the Gaussian density function. The weights correspond to the probabilities of the models:

$$
\begin{aligned}
\hat{\boldsymbol{x}}_{k} & =\sum_{i=1}^{N} \mu_{k}^{i} \hat{\boldsymbol{x}}_{k}^{i} \\
\boldsymbol{P}_{k} & =\sum_{i=1}^{N} \mu_{k}^{i} \boldsymbol{P}_{k}^{i}\left(\hat{\boldsymbol{x}}_{k}^{i}-\hat{\boldsymbol{x}}_{k}\right)\left(\hat{\boldsymbol{x}}_{k}^{i}-\hat{\boldsymbol{x}}_{k}\right)^{T}
\end{aligned}
$$

\section{GUIDANCE MODE IDENTIFICATION ALGORITHM}

In this paper, the generic EKF-IMM algorithm presented in Section II-B has been adapted to identify the guidance modes of an aircraft during the descent phase. In order to accomplish that, several models have been defined, each one 
corresponding to a different guidance mode. In Section III-A, the features common to all the model-matched EKF are presented. Section III-B shows the particularities of each model and how they are related to the selected guidance modes.

\section{A. Common part of the models}

Each model-matched EKF consists of a dynamics model (Eq. (9a)) and a measurement model (Eq. (9b)). Next, the form of these models common to all EKFs is presented.

1) Dynamics model: In this paper, the gamma-command model has been used to represent the aircraft dynamics. However, an extended state vector has been considered, which includes the temperature and the pressure: $\boldsymbol{x}=[v, h, m, \tau, p]$.

Atmospheric variables are required in order to express the measurements vector $\boldsymbol{y}$ in terms of $\boldsymbol{x}$ (see Eq. (9b)). To the best of our knowledge, all previous works satisfied this requirement by obtaining $\tau$ and $p$ from $h$ assuming the ISA.

The dynamics of $h, v$ and $m$ are particularsided by Eq. (1), while the following atmospheric model has been adopted to describe the differential equations of $\tau$ and $p$ :

$$
\frac{\mathrm{d} \tau}{\mathrm{d} t}=\tau_{h}(h) \frac{\mathrm{d} h}{\mathrm{~d} t} ; \quad \frac{\mathrm{d} p}{\mathrm{~d} t}=p_{h}(\tau, p) \frac{\mathrm{d} h}{\mathrm{~d} t}
$$

where $\tau_{h}$ and $p_{h}$ are the temperature and pressure partial derivatives with respect to altitude, respectively. In the troposphere, $\tau_{h}$ is set to a constant value according to the ISA temperature lapse rate. In hydrostatic equilibrium $p_{h}=-\rho g$ regardless of the aircraft altitude, being $\rho=p / R \tau$ the density of the air and $R$ the specific gas constant for dry air.

The BADA version 4.1 aircraft performance model has been used to define $T_{\max }, T_{\min }, D$ and $F F$ functions.

The initial state vector $\boldsymbol{x}_{0}$ is straightforward to be determined from flight and weather data except for the mass, which is not known and is not expected to be shared by the airlines. The algorithm proposed in this paper initially assumes a "guess" for the initial aircraft mass $\left(m_{0}\right)$, which is set to the $90 \%$ of the maximum landing mass as published in the BADA model. For those modes in which the throttle controls the thrust (i.e. the second degree of freedom is closed by Eq .(3)), $m$ can be inferred based on the observed temporal evolution of the specific energy of the aircraft: in such circumstances the power generated by the forces is known, and an "equivalent" mass can be found such that the specific power matches the observed specific energy rate. Several approaches have been proposed in the recent years to estimate the aircraft mass using this principle [17]. In this paper, the least-squares approach proposed in [17] has been adopted when the estimated guidance mode is such that the thrust is known during more than 30 consecutive seconds.

The vector of process noise is $\boldsymbol{q}=\left[0_{1 \times 3}, w_{\tau}, w_{p}\right]$, where $0_{n \times m}$ is a $n \times m$ matrix of zeros and $w_{i}(i \in\{\tau, p\})$ are process noise terms statistically independent from each other, used to account for non-standard atmospheric conditions. Those values have been set based on the expected deviation from the standard atmospheric conditions. Finally, the initial error covariance $\boldsymbol{P}_{0}$ has been defined based on the initialisation uncertainty: if $\boldsymbol{x}_{0}$ is not close to the real state $\boldsymbol{P}_{0}$ should be large, whereas if there is high confidence on $\boldsymbol{x}_{0}$ a lower value of $\boldsymbol{P}_{0}$ can be set.

2) Measurement model: Ground-based Mode S secondary surveillance radar (SSR) establishes selective and addressed interrogations with aircraft is sight aiming to track on-board transponders more precisely and more efficiently. The enhanced surveillance (EHS) level of the Mode $S$ is intended to improve the situational awareness of ATC by providing additional information about the state and intent of the aircraft.

On request by the radar, aircraft sends elements of information (called downlink aircraft parameters, DAPs) that include the current true airspeed, the Mach number, the indicated airspeed and the vertical rate (among others). In addition, through the Mode $\mathrm{S}$ extended squitter function used by ADSB systems, aircraft are able to transmit their position, velocity vector and altitude with a high emission rate (typically $1 \mathrm{~s}$ ).

In this paper, the observations gathered from Mode $\mathrm{S}$ and ADS-B data compose the measurements vector $\boldsymbol{y}=$ $\left[v, h_{p}, \dot{h}, \mathrm{VS}, v_{C A S}, M\right]$, which can be expressed in terms of $\boldsymbol{x}$ and $\boldsymbol{p}$. Observations of $v_{C A S}, M, \dot{h}$ and VS are collected from Comm-B Data Selector 60 (BDS60) EHS messages, $v$ from BDS50 messages and $h_{p}$ from ADS-B position reports.

Finally, $\boldsymbol{R}$ has been configured as a diagonal matrix (i.e. the measurement noise terms are assumed to be statically independent from each other) whose elements have been selected by trial and error and educated guesses.

\section{B. Specific guidance models}

As stated in Section II-B, the IMM algorithm works by defining a set of candidate models for the system rather than considering a unique model to unambiguously describe its behavior. In this paper, 13 different models have been defined, each one corresponding to a specific guidance mode and denoted by the two variables being controlled. The former term indicates the variable controlled by the elevator, the latter that controlled by the throttle. Table I shows the 13 considered models, which have been classified into three different sets.

The first set includes those models in which the acceleration is controlled by the elevator and the throttle maintains a given thrust value. For the second set, the acceleration is controlled by the throttle and the elevator keeps either the desired VS or ground FPA. Finally, the third set is composed by those guidance modes in which the elevator controls either VS or the ground FPA and the throttle is fixed to a certain value.

Each model-matched filter is complemented by choosing two of the three path constraints (see Section II-A) and the values for their associated parameters. The first path constraint corresponds to the elevator, and the second to the throttle.

The IMM algorithm proposed herein assumes that the parameters of each model take known and fixed values set by the user based on some prior knowledge. The values of the energy share factor during typical deceleration and acceleration phases (DEC and ACC) have been obtained from BADA [5]. The vertical speed has been chosen based the values specified by the flight crew operating manual (FCOM) 
TABLE I

DESCENT GUIDANCE MODE MODELS AND ASSOCIATED PARAMETERS

\begin{tabular}{|c|c|c|c|}
\hline Set & M & Elevator & Throttle \\
\hline \multirow{4}{*}{1} & MACH-THR & $k=\left.k\right|_{\dot{M}=0.0}$ & $\pi=0.0$ \\
\hline & CAS-THR & $k=\left.k\right|_{\dot{v}_{C A S}}=0.0$ & $\pi=0.0$ \\
\hline & DEC-THR & $k=0.3$ & $\pi=0.0$ \\
\hline & ACC-THR & $k=1.7$ & $\pi=0.0$ \\
\hline \multirow{9}{*}{2} & VS-MACH & $k=\left.k\right|_{\dot{M}=0}$ & $\mathrm{VS}=-1000 \mathrm{ft} \min ^{-1}$ \\
\hline & VS-CAS & $k=\left.k\right|_{\dot{v}_{C A S}=0.0}$ & $\mathrm{VS}=-1000 \mathrm{ft} \min ^{-1}$ \\
\hline & VS-DEC & $k=0.3$ & $\mathrm{VS}=-1000 \mathrm{ft} \min ^{-1}$ \\
\hline & VS-ACC & $k=1.7$ & $\mathrm{VS}=-1000 \mathrm{ft} \min ^{-1}$ \\
\hline & FPA-MACH & $k=\left.k\right|_{\dot{M}=0.0}$ & $\gamma_{g}=-3.0^{\circ}$ \\
\hline & FPA-CAS & $k=\left.k\right|_{\dot{v}_{C A S}}=0.0$ & $\gamma_{g}=-3.0^{\circ}$ \\
\hline & FPA-DEC & $k=0.3$ & $\gamma_{g}=-3.0^{\circ}$ \\
\hline & FPA-ACC & $k=1.7$ & $\gamma_{g}=-3.0^{\circ}$ \\
\hline & ALT-SPEED & $k=0.0$ & $\mathrm{VS}=0.0$ \\
\hline \multirow{3}{*}{3} & VS-THR & $\mathrm{VS}=-1000 \mathrm{ft} \mathrm{min}^{-1}$ & $\pi=0.0$ \\
\hline & FPA-THR & $\gamma_{g}=-3.0^{\circ}$ & $\pi=0.0$ \\
\hline & ALT-THR & $\mathrm{VS}=0.0$ & $\pi=0.0$ \\
\hline
\end{tabular}

of the A320 during early descents and repressurisation segments [18]. Finally, the geometric flight path angle has been set to a typical instrumental landing system (ILS) glide slope.

It could seem a good idea to define the parameters as augmented states aiming to improve the flexibility of the algorithm to adapt to practical scenarios, where the parameters will not take known values. It is not. When designing an IMM algorithm it is important to define the models of $\mathcal{M}$ as distinct as possible. Otherwise, all the models could be equally probable by adapting their parameters, and the algorithm would encounter difficulties to discriminate the true model.

Last but not least, the overall estimate of the IMM algorithm (as a result of the combination step) is a weighted sum of the estimates computed by each individual filter, with the weights based on the model probabilities. Therefore, even if any of the models defined in $\mathcal{M}$ does not perfectly match the real system behavior, the algorithm is still capable to perform accurate estimates using individual features of all the models.

Summing up, choosing fixed values for the model parameters it is not a rough assumption, but a requirement of the IMM algorithm design to achieve good performance.

\section{RESUlTS}

Firstly, this section shows the validation results of the algorithm presented above, where the Airbus Performance Engineering Program (PEP) has been used to synthesise two trajectories with a known sequence of guidance modes. Then, Section IV-B shows an application example where the guidance modes are identified from ADS-B and Mode S messages.

\section{A. Algorithm validation}

The Operational Flight Path (OFP) module of PEP allows to generate realistic trajectories using accurate performance data from the manufacturer. A trajectory generated with OFP is divided in several flight phases, each one expressed in terms
TABLE II

FLIGHT-INTENTS FOR THE VALIDATION TRAJECTORIES

\begin{tabular}{|c|c|c|c|c|}
\hline \multirow{2}{*}{ Phase } & \multicolumn{2}{|c|}{ M } & \multicolumn{2}{|c|}{ End condition } \\
\hline & VT1 & VT2 & VT1 & VT2 \\
\hline 1 & \multicolumn{2}{|c|}{ ALT-SPEED } & \multicolumn{2}{|c|}{$\Delta s=20 \mathrm{NM}($ distance $)$} \\
\hline 2 & VS-CAS & MACH-THR & $h_{p}=\mathrm{FL} 280$ & $v_{C A S}=300 \mathrm{kt}$ \\
\hline 3 & \multirow{2}{*}{\multicolumn{2}{|c|}{$\begin{array}{l}\text { CAS-THR } \\
\text { DEC-THR }\end{array}$}} & \multicolumn{2}{|c|}{$h_{p}=$ FL110 } \\
\hline 4 & & & $v_{C A S}=230 \mathrm{k}$ & $v_{C A S}=250 \mathrm{kt}$ \\
\hline 5 & CAS-THR & FPA-CAS & \multicolumn{2}{|c|}{$h_{p}=3000 \mathrm{ft}$} \\
\hline
\end{tabular}

of an initial condition, a parametrised guidance mode being targeted, and an end condition to be met.

The initial condition can be described as time, position, speed or altitude. Starting at the initial condition, the trajectory is numerically integrated according to the guidance mode, which sets commands for the throttle and the elevator until the end condition is met. Obviously, the end condition of a given phase corresponds to the initiation of the next one.

Flight data obtained from OFP contains the same information that could be gathered from ADS-B and Mode S messages, but are given every second and are not corrupted neither by measurement errors nor by random noise. Aiming to obtain meaningful results that could be extrapolated to real scenarios, OFP data has been randomly removed and Gaussian noise has been added to emulate realistic flight data. These perturbations $(\epsilon)$ are based on an analysis of the real data.

Given these flight data, the IMM filter has been used to identify the guidance modes of each validation trajectory (VT). Finally, the outcomes of the IMM filter have been analysed and compared with the known guidance modes sequence.

It should be noted that PEP uses accurate performance data from the manufacturer, while the proposed algorithm uses BADA 4.1 performance models. Therefore, the robustness of the algorithm to aircraft performance model-reality mismatches is implicitly assessed during this validation process.

An Airbus A320 has been selected as the aircraft for the validation process. The initial altitude for both VT is FL360. The first VT starts with a Mach number of 0.8 and an initial mass of $60,000 \mathrm{~kg}$. The initial Mach and mass for the second VT are 0.78 and 58,000, respectively. Table II shows the guidance mode and the end condition for each one of the flight phases of the VTs. The parameters of each guidance mode have been selected to match those defined in Table I.

The first VT represents a typical early descent, in which the ATC requests to descent well before the aircraft reaches the optimal top of descent (TOD). In these circumstances, the aircraft starts to descent at a constant VS controlled by the elevator, aiming to intercept the initially planned path from below whilst maintaining the planned speed with the throttle.

The second VT represents a nominal descent with a CAS/Mach speed schedule at idle thrust until reaching a waypoint with an associated altitude constraint. Thereafter, the elevator controls the FPA in the remaining geometric segments and the throttle is managed to follow the speed down to the instrumental landing system glide slope interception. 




(a) Early descent (VT1)

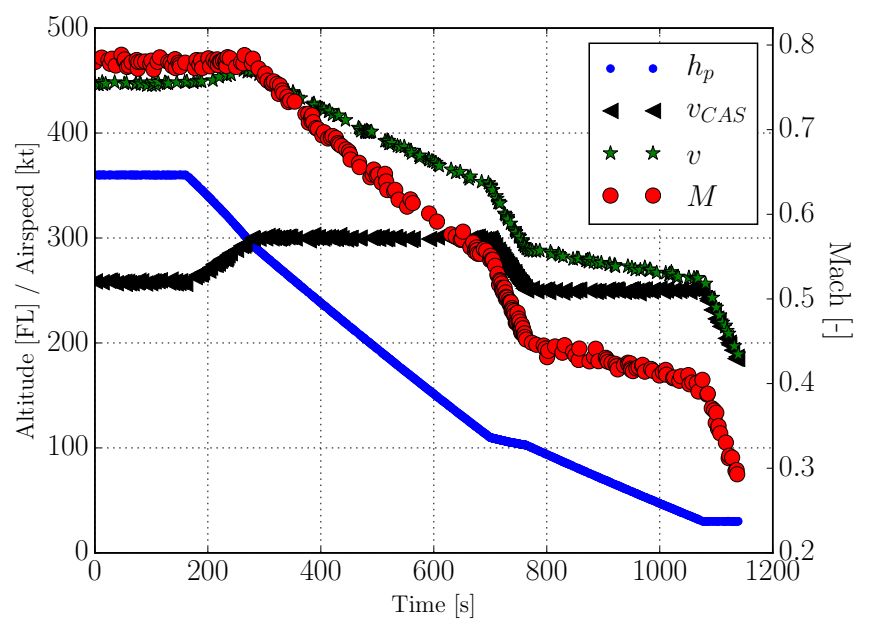

(b) Nominal descent (VT2)

Fig. 2. Validation trajectories

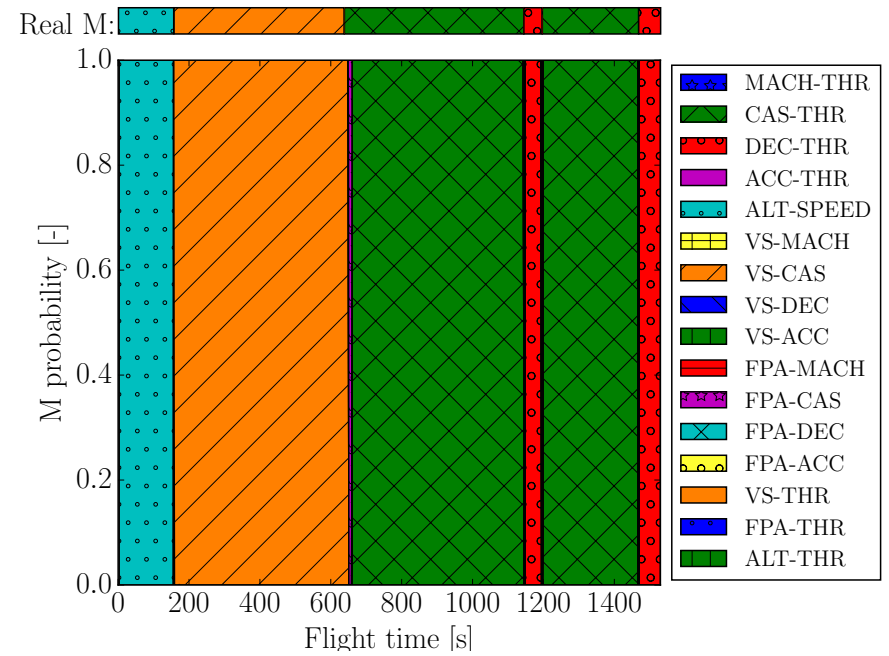

(a) Early descent (VT1)

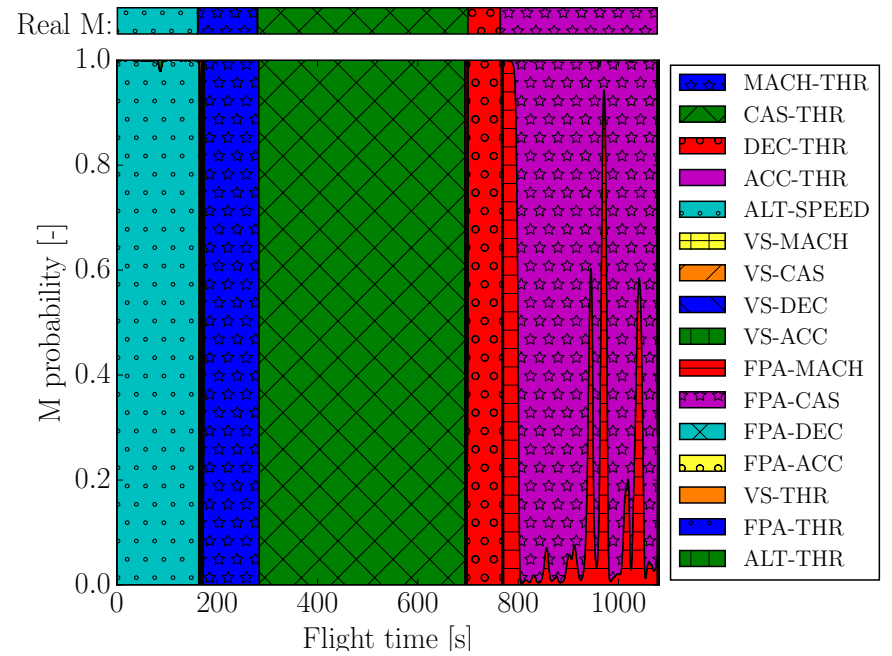

(b) Nominal descent (VT2)

Fig. 3. Model probabilities for the validation trajectories

Figures 2(a) and 2(b) show the emulated flight data for the first and second VTs, including the altitude and speed observations.

Fig. 3 shows the real and the predicted model probabilities as a function of the time for the two VTs. Results show that the proposed algorithm is able to unequivocally identify the actual guidance mode. In addition, few observations are required to identify a guidance mode change, as demonstrated by the insignificant delay between the real and the predicted guidance mode switching instants.

It is interesting to note that, approximately at 790 seconds, the nominal VT2 (Fig. 3(b)) switches from DEC-THR to FPACAS. However, the algorithm predicts a FPA-MACH guidance mode due to the noise in the measurements and the similarity between these phases in terms of kinetic and potential energy rates. After few steps of the algorithm, however, the likelihood of the FPA-CAS model becomes quickly to almost 1 . From that time on, the probability of FPA-MACH show intermittent increases, but almost never overtakes that of FPA-CAS.

At each time step, the identified guidance mode has been used to predict the future short-term state of the aircraft, assuming that the guidance mode remains unchanged. Then, the predicted altitude and speed have been compared with those of the real trajectory to assess the prediction errors. Figure 4 shows the prediction errors as a function of the lookahead time for the nominal VT2. The analogous results for the VT1 are similar and would not give additional information.

One of the limitations of the proposed algorithm is the lack of a mechanism to predict the end condition of the active flight phase (i.e. the switching condition from a guidance mode to the next one). That is, the short-term state prediction is computed assuming that the aircraft will not modify its behavior, leading to significant errors if the guidance mode 


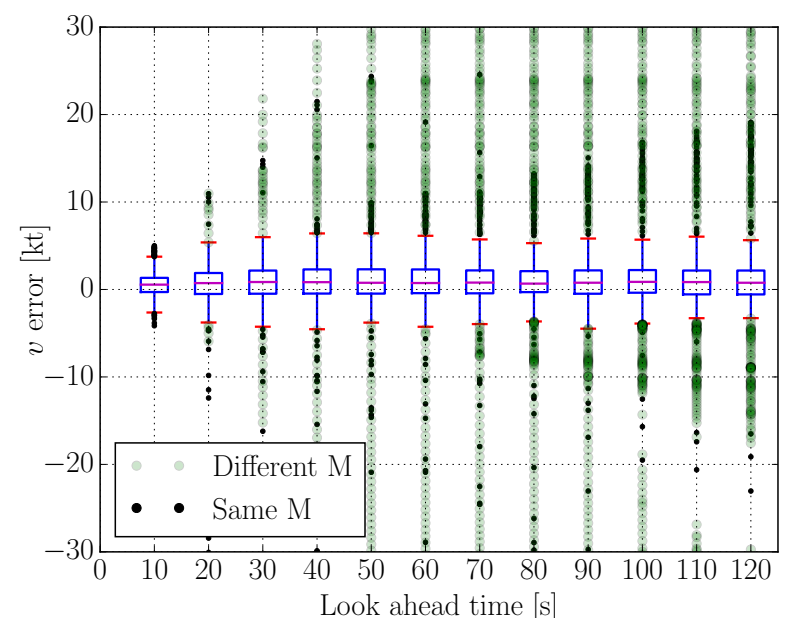

(a) Speed

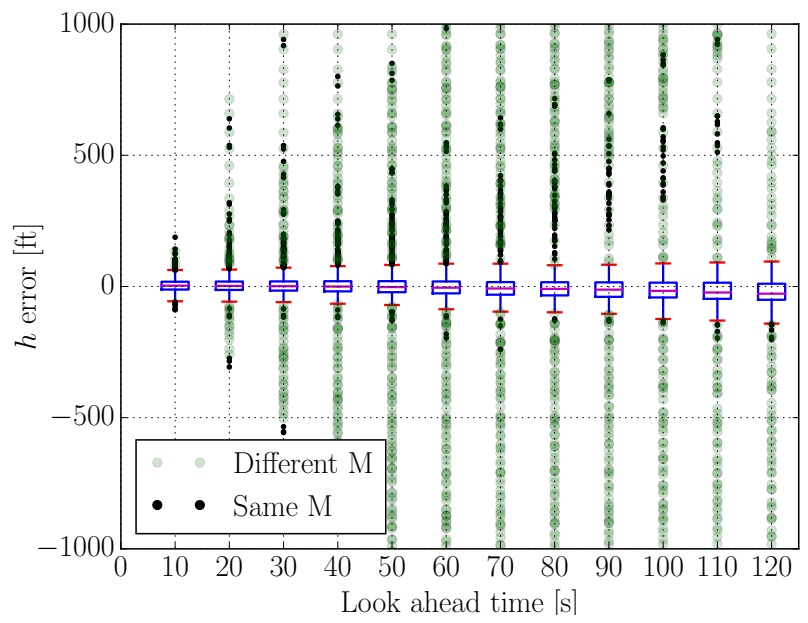

(b) Altitude

Fig. 4. Prediction errors for the validation trajectories

changes unanticipatedly. The short-term predictions could be complemented with knowledge about the aircraft intent to estimate the end condition of the current phase. This information could be given by the pilot, taken from ARPM, estimated from ATM constraints or derived from issued ATC instructions.

The box-and-whiskers plots shown in Fig. 4 have been generated for such predictions in which the current and the lookahead prediction time belong to the same guidance mode (i.e. they present the best-case results, assuming an hypothetical scenario in which the end condition of the phase is known).

In Fig. 4, the bottom and top of the box represent the first and third quartiles, respectively. The lines extending vertically from the boxes (whiskers) indicate variability outside these quartiles, while the ends of the whiskers represent the 1.5 interquartile range (IQR) of the first and third quartile. Finally, the band inside the box is the median and outliers are represented as circles. The additional green circles show the prediction errors that would result if the end condition of the current phase were not known. I should be noted that since the outliers lie outside the 1.5 IQR of the first and third quartiles, they represent a very small fraction of the total samples.

As a final remark, each tic in Fig. 4 includes the aggregated prediction errors of all the predictions computed along the trajectory for the corresponding look-ahead time.

As expected, both median and variability of the altitude prediction error tend to increase with the look-ahead prediction time. Surprisingly, these parameters for the speed prediction error remain constant (or slowly increase) with the look-ahead prediction time. Finally, the predicted speed and altitude errors after 2 minutes are expected to be lower than $5 \mathrm{kt}$ and 100 $\mathrm{ft}$ for most of the cases, respectively, provided that the end on the current phase is known. Otherwise, the green circles evidence that large prediction errors could be obtained.

It should be noted that these results were obtained only for two particular VTs and therefore cannot be generalised.
Further analysis with more VTs generated with OFP showed that, under certain circumstances, several of the models defined in Table I could be very similar in terms of kinetic and potential energy rates (e.g. FPA-CAS and FPA-MACH at low altitudes). In such cases, the IMM algorithm may encounter difficulties to discriminate the "true" guidance mode, continuously switching between potential models. This behavior shall not deteriorate the prediction of future states, provided that the candidate models effectively represent the real behavior of the aircraft. However, a smoothing process of the models probabilities may be required if the sequence of guidance modes is intended to ATM analytics [19] .

\section{B. Identification of guidance modes of a real trajectory}

Next, the guidance modes along the trajectory of an Airbus A319 descending at Barcelona-El Prat are identified by using real flight data. These data have been collected the $27^{\text {th }}$ December of 2016 with a receiver located nearby the airport.

At the lower part of the descent, before intercepting the ILS glide slope, aircraft start configuring with flaps according to a manufacturer-prescribed schedule that defines safe flap surface exposure as a function of aircraft airspeed. In practice, however, the exact moment at which flaps are extended and the landing gear is deployed depends on the particular circumstances and the pilot criteria. In absence of an algorithm capable of capturing changes of configuration, which significantly affect the aircraft performance, it was decided to stop the guidance mode identification algorithm at 3,000 ft.

Figure 5 shows the flight data collected for this flight. By comparing Figs. 2(a) and 5(a) it can be observed that the real flight own the main features of a typical early descent.

From 0 to 40 seconds the aircraft is still at the cruise phase, at constant altitude and Mach (ALT-SPEED). Suddenly, the aircraft starts to descent at constant vertical speed $\left(-1000 \mathrm{ft} \min ^{-1}\right)$ keeping the Mach number unchanged (VS$\mathrm{MACH})$. This maneouver could be caused either by an ATC 


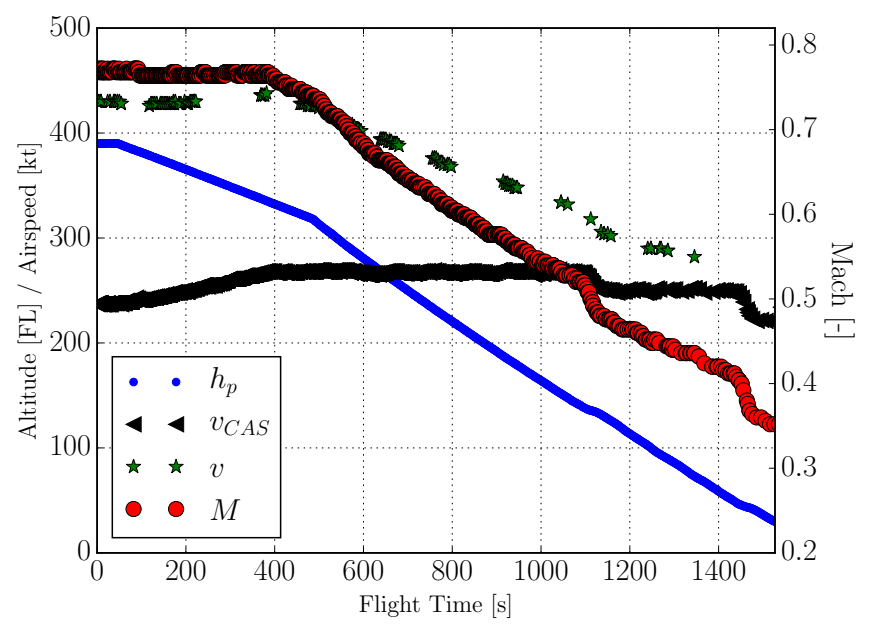

(a) Altitude and speed



(b) Vertical speed

Fig. 5. Real descent measurements (surveillance data)

instruction to start the descent immediately or by the need of a repressurisation segment. At the cross-over altitude (approximately FL320) the guidance mode switches from VS$\mathrm{MACH}$ to VS-CAS. Ninety seconds later, the planned path is intercepted from below, and the aircraft reduces the throttle to idle maintaining constant CAS down to FL130, where a small deceleration segment at idle thrust and constant energy share factor is performed (DEC-THR). This deceleration segment was probably performed to comply with the speed limit at FL100 (typically $250 \mathrm{kt}$ ) enforced by ATM regulations. The last part of the descent consists of a CAS-THR phase followed by another DEC-THR until reaching 3,000 ft.

It is interesting to note that the inertial VS, which is derived from navigation data sources in the aircraft avionics, is smoother than the barometric altitude rate.

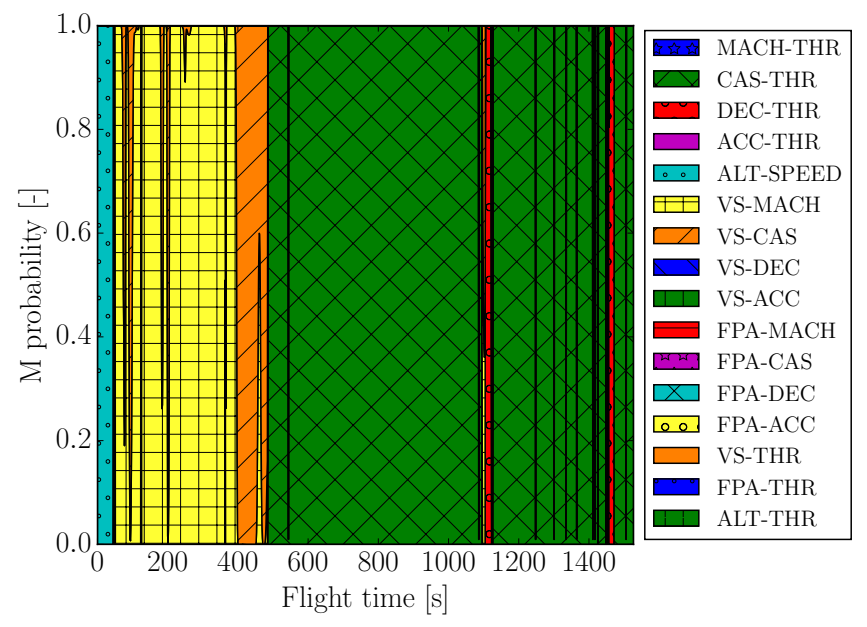

Fig. 6. Model probabilities for the real trajectory

Fig. 6 shows the IMM model probabilities. According to this, after the small cruise segment a VS-MACH phase is identified. Although at some time instants the probability of the VS-CAS model increases due to the noise in the measurements and the unmodelled tropopause, the proposed algorithm maintains a higher confidence level with VS-MACH.

Below FL360 (where the temperature gradient with altitude is well modelled), the algorithm confirms the VS-MACH with less hesitation. The cross-over altitude is identified with almost no delay, determining the switching condition from VS-MACH to VS-CAS. The following CAS-THR phase is perhaps the most evident from the algorithm point of view, with almost null probability set to the other guidance modes. Following, the two deceleration phases at idle thrust are also recognised at the right moment. Finally, it can be also observed that at some time instants of the second CAS-THR phase the IMM algorithm shows fleeting changes in the guidance mode, rapidly realising that the CAS-THR guidance mode is still active.

Figure 7 shows the analogous of Fig. 4 for the real trajectory. According to this Figure, for the real flight the median of both speed and altitude prediction errors increase (in absolute terms) with the look-ahead time, and almost no tendency of the error variability with the look-ahead time is observed. In deed, the patterns for the speed and altitude prediction errors are similar to those obtained for the early descent VT.

\section{Conclusions}

In this paper, an interacting multiple-model (IMM) algorithm, capable to identify the actual guidance mode of aircraft from Aircraft Dependent Surveillance-Broadcast (ADS-B) and selective mode (Mode S) flight data has been presented. Results showed that the actual guidance mode of an aircraft can be unambiguously identified in almost real time. In addition, short-time predictions using the estimated actual guidance mode showed acceptable speed and altitude prediction errors.

This algorithm could support a wide range of air traffic management (ATM) applications and tools that require accurate trajectory predictors and conflict detection and resolution 


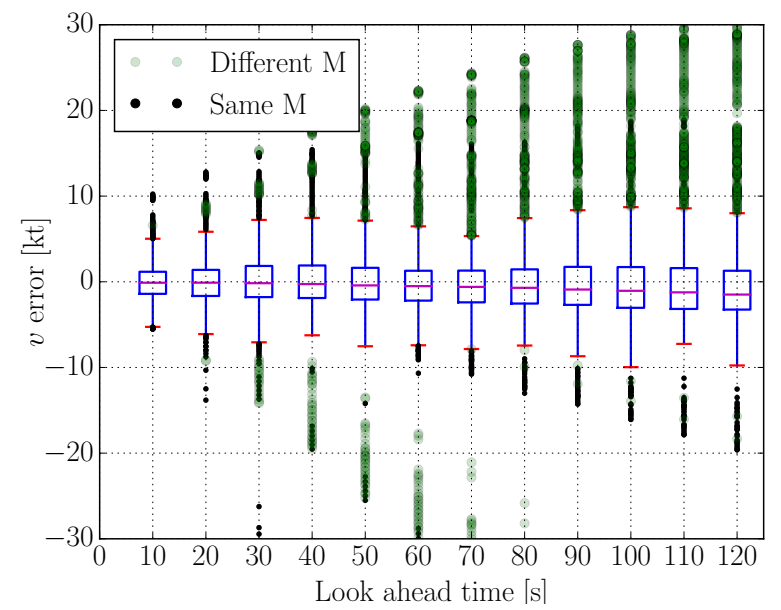

(a) Speed

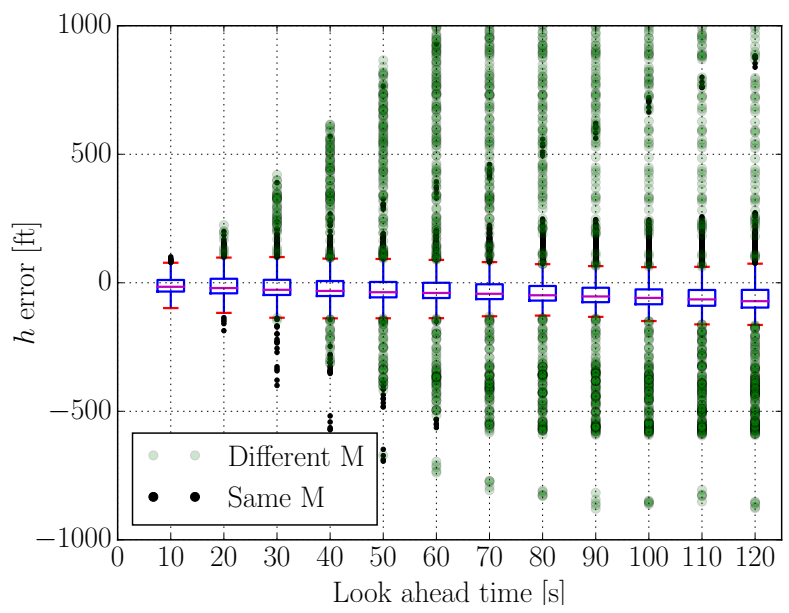

(b) Altitude

Fig. 7. Altitude prediction errors for the real trajectory

algorithms. Moreover, it could open the door to ATM analytics and air transportation performance monitoring or assessment.

The aim of this paper was to set the basis of the proposed algorithm and to demonstrate its potential capabilities. In future work, the algorithm will be tested against a larger set of trajectories and the most relevant performance indicators will be investigated (e.g. prediction errors, percentage of erroneous guidance mode estimations, guidance mode identification delay). In addition, it is expected to add the lateral dynamics (with the corresponding lateral models) and certain intelligence or heuristic to estimate the end of the active guidance mode. Moreover, the guidance mode models could be redefined to mach those from existing standards, such as the aircraft intent description language (AIDL) [20].

\section{ACKNOWLEDGMENTS}

The authors would like to thank Airbus Industries for the use of PEP (Performance Engineers Program) suite, which allowed us to undertake realistic aircraft performances simulations.

\section{REFERENCES}

[1] W. Schuster and W. Ochieng, "Performance requirements of future Trajectory Prediction and Conflict Detection and Resolution tools within SESAR and NextGen : Framework for the derivation and discussion," Journal of Air Transport Management, vol. 35, pp. 92-101, 2014.

[2] B. Strajnar, "Validation of Mode-S Meteorological Routine Air Report aircraft observations," Journal of Geophysical Research Atmospheres, vol. 117, no. 23, pp. 1-10, 2012.

[3] K. Legrand, C. Rabut, and D. Delahaye, "Wind networking applied to aircraft trajectory prediction," in 34th IEEE/AIAA Digital Avionics Systems Conference (DASC), Sacramento, USA, 2015.

[4] P. P. Duan, M. U. de Haag, and T. Etherington, "Energy state prediction methods for airplane state awareness," in IEEE/AIAA 35st Digital Avionics Systems Conference (DASC), Sacramento, USA, 2016.

[5] Eurocontrol, "User manual for the base of aircraft data ( $\{\mathrm{BADA}\})$. Revision 4.1," Bretigny (France), sep 2014.

[6] M. Hrastovec and F. Solina, "Prediction of aircraft performances based on data collected by air traffic control centers," Transportation Research Part C: Emerging Technologies, vol. 73, pp. 167-182, 2016.
[7] E. Mazor, A. Averbuch, Y. Bar-Shalom, and J. Dayan, "Methods in Target Tracking : A Survey," IEEE Transactions on Aerospace and Electronic Systems, vol. 34, no. 1, 1998.

[8] R. Li and V. P. Jilkovt, "A Survey of Maneuvering Target TrackingPart IV: Decision-Based Methods," in Proceedings of SPIE Conference on Signal and Data Processing of Small Targets, Orlando (USA), 2002.

[9] Q. Wang and J. Huang, "A VB-IMM filter for ADS-B data," in International Conference on Signal Processing Proceedings, ICSP, vol. 2015-Janua, no. October, 2015, pp. 2130-2134.

[10] H. Khadilkar and H. Balakrishnan, "A Multi-Modal Unscented Kalman Filter for Inference of Aircraft Position and Taxi Mode from Surface Surveillance Data," Proceedings of the AIAA Aviation Technology, Integration, and Operations Conference, pp. 1-11, 2011.

[11] D. Jeon, Y. Eun, H. Bang, and C. Yeom, "Nonlinear aircraft tracking filter utilizing a point mass flight dynamics model," Proceedings of the Institution of Mechanical Engineers, Part G: Journal of Aerospace Engineering, vol. 227, no. 11, pp. 1795-1810, 2013.

[12] J. L. Yepes, I. Hwang, and M. Rotea, "New Algorithms for Aircraft Intent Inference and Trajectory Prediction," Journal of Guidance, Control, and Dynamics, vol. 30, no. 2, pp. 370-382, mar 2007. [Online]. Available: http://dx.doi.org/10.2514/1.26750

[13] P. K. Menon, G. D. Sweriduk, and B. Sridhar, "Optimal Strategies for Free-Flight Air Traffic Conflict Resolution," Journal of Guidance, Control, and Dynamics, vol. 22, no. 2, 1999.

[14] J. Bronsvoort, "Contributions to trajectory prediction theory and its application to arrival management for air traffic control," 2014.

[15] H. A. P. Bloom, "An efficient filter for abruptly changing systems," in Decision and Control, 1984. The 23rd IEEE Conference on, no. December 1984, 1984, pp. 656 - 658.

[16] G. A. Einicke, Smoothing, filtering and prediction: Estimating the past, present and future. InTech, 2012.

[17] R. Alligier, D. Gianazza, and N. Durand, "Ground-based Estimation of the Aircraft Mass, Adaptive vs. Least Squares Method," in 10th USA/Europe Air Traffic Management Research and Developpment Seminar, vol. 2013, no. June, Chicago, Illinois USA, 2013.

[18] Airbus, "Flight Crew Operation Manual (FCOM). A320. Version 1.3.1," 1993.

[19] S. Särkkä, P. Bunch, and S. J. Godsill, "A backward-simulation based Rao-Blackwellized particle smoother for conditionally linear Gaussian models," IFAC Proceedings Volumes (IFAC-PapersOnline), vol. 16, no. PART 1, pp. 506-511, 2012.

[20] J. López-Leonés, M. A. Vilaplana, E. Gallo, F. A. Navarro, and C. Querejeta, "The aircraft intent description language: A key enabler for airground Synchronization in trajectory-based operations," in AIAA/IEEE Digital Avionics Systems Conference - Proceedings, 2007, pp. 1-12. 